Check for updates

Cite this: RSC Adv., 2019, 9, 26030

Received 20th June 2019

Accepted 14th August 2019

DOI: $10.1039 / c 9 r a 04645 c$

rsc.li/rsc-advances

\section{Role of electron in intramolecular vibrational energy redistribution: a simulation of time- and frequency-resolved CARS spectrum}

\author{
Zanhao Wang, (D) $\dagger^{a}$ Honglin Wu, (D) $\dagger^{b}$ Xiaosong Liu, (D) ${ }^{a}$ Yunfei Song ${ }^{b}$ \\ and Yanqiang Yang*ab
}

A coupled oscillator model with special attention to the electron is employed to simulate the time- and frequency-resolved coherent anti-Stokes Raman scattering (TFR-CARS) spectrum of benzene, where the electronic contribution is introduced as an oscillator as well as molecular vibration, and both the coupling between molecular vibrations and the coupling between electron and molecular vibration are involved. Through the simulation, the intramolecular vibrational energy redistribution (IVR) process is confirmed to occur more readily between the molecular vibrations with the same vibrational symmetry. Moreover, it is found that the electron plays a mediator role in the IVR process, and the coupling between electron and molecular vibration significantly increases the intramolecular vibrational energy transfer efficiency.

\section{Introduction}

Intramolecular vibrational energy redistribution (IVR) processes significantly influence the localization of vibrational energy in molecules. ${ }^{1}$ The determination of the paths of vibrational energy flow after a chemical bond is selectively excited, is very important for the control of chemical reactions. Several vibrational spectroscopic methods, such as, IR-Raman ${ }^{2-4}$ and coherent anti-Stokes Raman Scattering (CARS), ${ }^{5-7}$ have been established to track the IVR dynamics. With femtosecond timeand frequency-resolved CARS (TFR-CARS) technique, the vibrational modes can be coherently excited. ${ }^{8}$ The detection process consequently avoids the thermal population flow and will reflect the energy redistribution processes inside molecules.

Recently, TFR-CARS experiments have been performed in our laboratory to track the coherent intramolecular vibrational energy transfer in liquid benzene ${ }^{9}$ and a series of structurally relevant monosubstituted benzenes. ${ }^{10}$ Take the experimental results of liquid benzene for instance, when the $\mathrm{C}-\mathrm{H}$ stretching vibrational modes of benzene at about $3000 \mathrm{~cm}^{-1}$ (parent modes) were selectively excited, vibrational modes around $1000 \mathrm{~cm}^{-1}$ (daughter modes) far away from the direct excitation region were observed. The experimental results manifest the existence of IVR and the practicability of tracking IVR by CARS.

${ }^{a}$ Department of Physics, Harbin Institute of Technology, Harbin, 150001, P. R. China. E-mail: yqyang@hit.edu.cn

${ }^{b}$ National Key Laboratory of Shock Wave and Detonation Physics, Institute of Fluid Physics, China Academy of Engineering Physics, Mianyang, 621900, P. R. China

$\dagger$ These authors contributed equally.
However, only qualitative interpretation was given in the previous work. In order to get an insight into the physical mechanism of the observed IVR processes, further theoretical analyses are necessary.

The theories of TFR-CARS have been proposed for a long time involving both classical ${ }^{11,12}$ and quantum ${ }^{13,14}$ approaches. No matter which approach was used, for simplification, the vibrational modes were usually assumed to be independent, which means that the simulated CARS spectrum will not display the energy transfer process between different vibrational modes. When the IVR process is concerned, the coupling between different modes should be involved into the theoretical models. Besides, in CARS spectra, the electronic contribution was always considered as the non-resonant (NR) background, ${ }^{15}$ which was usually treated as a Gaussian envelope in time domain and simply added with the simulated vibrational dynamics. However, the properties of a molecular system are determined by the motion of the nuclei and electrons and the interaction between them, hence the electronic contribution to CARS spectrum was always underestimated in previous theories.

In this work, the electronic contribution is paid special attention to. Electron is considered as oscillator and introduced to the oscillator model for simulating TFR-CARS. The electronvibration coupling and the vibration-vibration coupling are simultaneously considered in our model. The theoretical simulation based on this coupled oscillator model is carried out, taking the CARS of benzene as the object, to acquire the respective contribution from electron and molecular vibration and the related coupling effects. 


\section{Theoretical basis}

A molecule is composed of atomic cores and valence electrons, and here, we assume that the molecular vibration is dominated by the motion of atomic cores, and the electronic vibration is dominated by the motion of valence electrons. Both the molecular vibration and the electronic vibration are considered as oscillators. ${ }^{\mathbf{1 6}}$ The quadratic couplings are considered between different vibrational modes, and the interaction between electron and molecular vibrational mode is described by the Coulomb interaction. Involving the interaction with the electric field component of light, the Hamiltonian can be written as

$$
\begin{aligned}
H= & \sum_{\mathrm{v}}\left(\frac{p_{\mathrm{v}}{ }^{2}}{2 m_{\mathrm{v}}}+\frac{1}{2} m_{\mathrm{v}} \omega_{\mathrm{v}}{ }^{2} q_{\mathrm{v}}^{2}\right)+\sum_{\mathrm{v}^{\prime} \neq \mathrm{v}} K_{\mathrm{vv}^{\prime}} q_{\mathrm{v}} q_{\mathrm{v}^{\prime}} \\
& +\sum_{\mathrm{v}} \frac{\partial \alpha_{\mathrm{v}}}{\partial q_{\mathrm{v}}} q_{\mathrm{v}} E_{\mathrm{pu}}(t) E_{\mathrm{st}}^{*}(t)+\frac{p_{\mathrm{e}}^{2}}{2 m_{\mathrm{e}}}+\frac{1}{2} m_{\mathrm{e}} \omega_{\mathrm{e}}^{2} q_{\mathrm{v}}^{2}+\sum_{\mathrm{v}} K_{\mathrm{ve}} q_{\mathrm{v}} q_{\mathrm{e}} \\
& +\frac{\partial \alpha_{\mathrm{e}}}{\partial q_{\mathrm{e}}} q_{\mathrm{e}} E_{\mathrm{pu}}(t) E_{\mathrm{st}}^{*}(t) .
\end{aligned}
$$

Here, the subscript $\mathrm{v}$ and e represent vibrational modes and electron, respectively. $q$ and $p$ are canonical variables which represent the coordinate and momenta of oscillator. $m, \omega$ and $\alpha$ are respectively the normal coordinate, reduced mass, frequency and polarizability of each oscillator. $K_{i j}$ represents the coupling strength between the $i$ th and $j$ th oscillator. $E_{\mathrm{pu}}$ and $E_{\mathrm{st}}$ are the electric field strengths of pump light and Stokes light. Together with $E_{\mathrm{pr}}$ of the probe light, all the light pulses are treated as transform limited Gaussian pulses and have the form as

$$
E_{i}(t)=E_{i}^{0} \exp \left(-2 \ln 2 \frac{t^{2}}{\Delta_{i}^{2}}\right) \exp \left(-i \omega_{i} t\right),
$$

where each pulse has carrier frequency $\omega_{i}$, and has the pulse duration $\Delta_{i}$ given as the full width at half maximum (FWHM) of the laser pulse.

The ensemble averaged coherent vibrational excitation of the molecule in the CARS experiment is described by coherence amplitude $q$, the expectation value of displacement, which contains the amplitude and phase. Using the Hamilton's equations, ${ }^{17}$ the following coupled difference equations for the Raman-active vibrational modes and electron are obtained

$$
\begin{aligned}
& \left(m_{\mathrm{v}} \frac{\mathrm{d}^{2}\left\langle q_{\mathrm{v}}\right\rangle}{\mathrm{d} t^{2}}+2 \frac{m_{\mathrm{v}}}{T_{2 \mathrm{v}}} \frac{\mathrm{d}\left\langle q_{\mathrm{v}}\right\rangle}{\mathrm{d} t}+m_{\mathrm{v}} \omega_{\mathrm{v}}{ }^{2}\left\langle q_{\mathrm{v}}\right\rangle+\sum_{\mathrm{v}^{\prime}, \mathrm{v}^{\prime} \neq \mathrm{v}} K_{\mathrm{vv}^{\prime}}\left\langle q_{\mathrm{v}^{\prime}}\right\rangle+K_{\mathrm{ve}}\left\langle q_{\mathrm{e}}\right\rangle\right. \\
& =\frac{\partial \alpha_{\mathrm{v}}}{\partial q_{\mathrm{v}}} E_{\mathrm{pu}} E_{\mathrm{st}}^{*} m_{\mathrm{e}} \frac{\mathrm{d}^{2}\left\langle q_{\mathrm{e}}\right\rangle}{\mathrm{d} t^{2}}+2 \frac{m_{\mathrm{e}}}{T_{2 \mathrm{e}}} \frac{\mathrm{d}\left\langle q_{\mathrm{e}}\right\rangle}{\mathrm{d} t}+m_{\mathrm{e}} \omega_{\mathrm{e}}{ }^{2}\left\langle q_{\mathrm{e}}\right\rangle+\sum_{\mathrm{v}} K_{\mathrm{ve}}\left\langle q_{\mathrm{v}}\right\rangle \\
& =\frac{\partial \alpha_{\mathrm{e}}}{\partial q_{\mathrm{e}}} E_{\mathrm{pu}} E_{\mathrm{st}}^{*}
\end{aligned}
$$

The damping of the molecular vibration is phenomenologically involved as the second terms in the equations of motion, where $T_{2}$ is the dephasing time. Then, eqn (3) can be numerically solved with the four-order Runge-Kutta-Merson method. ${ }^{18}$

In CARS process, the coherence amplitude $\langle q\rangle$ produced by the driving force of synchronous pump and Stokes laser pair is detected by a delayed probe pulse, and the corresponding thirdorder polarization is

$$
P^{(3)}(t, \tau)=\left[\sum_{\mathrm{v}} \lambda_{\mathrm{v}}\left\langle q_{\mathrm{v}}(t)\right\rangle+\lambda_{\mathrm{e}}\left\langle q_{\mathrm{e}}(t)\right\rangle\right] E_{\mathrm{pr}}(t-\tau),
$$

where $\lambda$ contains the cross-section of oscillators. The thirdorder polarization finally generates the femtosecond TFRCARS signal, which can be recorded as a function of frequency $\omega_{\mathrm{s}}$ and the delay time $\tau$ between the probe pulse and the simultaneous pump and Stokes pulses,

$$
S_{\mathrm{CARS}}\left(\omega_{\mathrm{s}}, \tau\right) \propto\left|\frac{1}{\sqrt{2 \pi}} \int_{0}^{+\infty} P^{(3)}(t, \tau) \exp \left(-i \omega_{\tau} t\right) \mathrm{d} t\right|^{2} .
$$

The corresponding Fourier transform (FT) spectrum of CARS can be obtained by performing Fourier transformation along the delay time $\tau$ axis

$$
S_{\mathrm{CARS}}\left(\omega_{\mathrm{s}}, \omega_{\tau}\right)=\frac{1}{\sqrt{2 \pi}} \int_{0}^{+\infty} S_{\mathrm{CARS}}\left(\omega_{\mathrm{s}}, \tau\right) \exp \left(-i \omega_{\tau} \tau\right) \mathrm{d} \tau .
$$

The FT spectrum of CARS will be used to analyze the coherent coupling between the relevant vibrations.

It should be noted that the electronic contribution, which typically dominates only when the three incident laser pulses (Raman pump, Stokes and probe pulses) overlap temporally, is naturally involved into the CARS spectrum by introducing electronic oscillator into eqn (3).

\section{Results}

\subsection{Simulation configuration}

The simulation parameters are set according to the experimental results in ref. 9. In this previous experimental work, five fundamental modes, labelled as $v_{1-5}$, have been identified participating in the IVR process. The two parent modes around $3000 \mathrm{~cm}^{-1}\left(v_{1}\right.$ and $\left.v_{2}\right)$ were directly excited, and the three daughter modes around $1000 \mathrm{~cm}^{-1}\left(v_{3-5}\right)$ emerged due to the vibrational energy transfer from parent modes. A directly excited combination mode at about $3165 \mathrm{~cm}^{-1}$, labelled as $v_{6}$, was identified as $2 v_{4}+v_{3}$. This combination mode did not participate in the IVR process, but contributed to the polarization beats in the high-frequency region. Besides, electron is also considered as an oscillator $\left(v_{\mathrm{e}}\right)$, with the oscillating frequency set to be $39677 \mathrm{~cm}^{-1}$ corresponding to the transition frequency from the electronic ground state to the first excited electronic singlet state $\mathrm{S}_{1} \cdot{ }^{19}$ The reduced mass of electron is set to be 0.023 , and all the reduced masses of these vibrational modes are got from the Gaussian calculation (using the density functional b3lyp and basis set 6-311g). ${ }^{20}$ 
Table 1 Information and parameters of the oscillators involved in the simulation

\begin{tabular}{|c|c|c|c|c|c|c|}
\hline & $v_{1}$ & $v_{2}$ & $v_{3}$ & $v_{4}$ & $v_{5}$ & $v_{6}$ \\
\hline Mode & $\mathrm{C}-\mathrm{H}$ stretching & C-H stretching & C-H bending & Ring breathing & C-H wagging & Combination mode \\
\hline Symmetry & $\mathrm{A}_{1 \mathrm{~g}}$ & $\mathrm{E}_{2 \mathrm{~g}}$ & $\mathrm{E}_{2 \mathrm{~g}}$ & $A_{1 g}$ & $\mathrm{E}_{1 \mathrm{~g}}$ & $\mathrm{E}_{2 \mathrm{~g}}$ \\
\hline Frequency/cm ${ }^{-1}$ & 3074 & 3056 & 1181 & 992 & 855 & 3165 \\
\hline Reduced mass/amu & 1.10 & 1.09 & 1.14 & 6.03 & 1.25 & 0.96 \\
\hline Dephasing time/ps & 1.50 & 1.50 & 0.50 & 0.50 & 0.80 & 1.50 \\
\hline
\end{tabular}

The dephasing time is determined from the experimental time-domain CARS spectrum. It is worth mentioning that both the energy of the incident light beams and the energy difference between any two laser beams are not resonant with the electron transition, then the electron is undergoing forced oscillation and the dephasing time is consequently very short, which is set to be $0.01 \mathrm{ps}$ in the simulation. All the relevant information is listed in Table 1.

The parameters of the incident laser pulses are set according to the experimental conditions, the central wavelengths of pump, Stokes and probe pulse are respectively set as $640 \mathrm{~nm}, 800 \mathrm{~nm}$ and $640 \mathrm{~nm}$, and the FWHMs of all pulses are set as $100 \mathrm{fs}$.

As mentioned above, the coupling effects between a pair of molecular vibrations and between molecular vibration and electron are both included, whose strengths are described by $K_{i j}$. In order to determine the coupling strengths, some qualitative relationship was summarized from the experimental results. Firstly, coherent vibrational energy transfer is more likely to occur between vibrational modes with the same symmetry, resulting in a larger coupling strength. Secondly, the role of electron in the IVR process cannot be ignored. In the present work, all the coupling strength will be used as adjustable parameters in the simulation process. Through reproducing the experimental spectrum by simulation, the coupling strength between different modes (including electron and vibrations) can be quantitatively determined.

\subsection{Simulation results}

(a) Spectra in time domain. For convenience of parameter adjustment, we introduce $\varpi_{i j}$ which has the same dimension of wavenumber as oscillator's frequency, and it relates to the coupling strength by $K_{i j}=\sqrt{m_{i} m_{j}} \varpi_{i j}{ }^{2}$. Here, the subscript numbers are corresponding to the vibrational modes listed in Table 1. To get the best simulation result, the couplings between different oscillators are listed in Table 2. It should be mentioned that only three couplings between vibrational modes are set to be nonzero, which are adopted corresponding

Table 2 The coupling strength between different oscillators obtained from the simulation, the unit of $\mathrm{cm}^{-1}$. Here, $K_{i j}=\sqrt{m_{i} m_{j}} \varpi_{i j}{ }^{2}$, refer to Table 4

\begin{tabular}{llllllll}
\hline$\varpi_{14}$ & $\varpi_{23}$ & $\varpi_{45}$ & $\varpi_{\mathrm{e} 1}$ & $\varpi_{\mathrm{e} 2}$ & $\varpi_{\mathrm{e} 3}$ & $\varpi_{\mathrm{e} 4}$ & $\varpi_{\mathrm{e} 5}$ \\
\hline 400 & 325 & 220 & 600 & 350 & 940 & 1200 & 100
\end{tabular}

to the conjecture in ref. 9. The physical meanings of these coupling strengths will be discussed in the Discussion section. The experimental CARS spectrum of benzene is given in Fig. 1(a). The corresponding simulated CARS spectrum of benzene, as shown in Fig. 1(b), is obtained by successively solving eqn (3)-(5) with the parameters given in Tables 1 and 2.

By comparing Fig. 1(a) and (b), it can be seen that the simulated spectrum reproduces the main features of the experimental result. For example, there is a slowly damped oscillating signal around $3000 \mathrm{~cm}^{-1}$ after $\sim 0.2 \mathrm{ps,} \mathrm{which}$ mainly arises from the interference of $v_{2}$ and $v_{6}$. Besides, a weak and quickly decayed signal emerges in the region around $1000 \mathrm{~cm}^{-1}$ far away from the directly excitation region, which was assigned to be a result of IVR from parent modes. Moreover, a NR background signal several orders higher than the damped signal also appears, which mainly originates from the electronic contribution. Both of NR electronic contribution and the interference of involved molecular vibrational modes
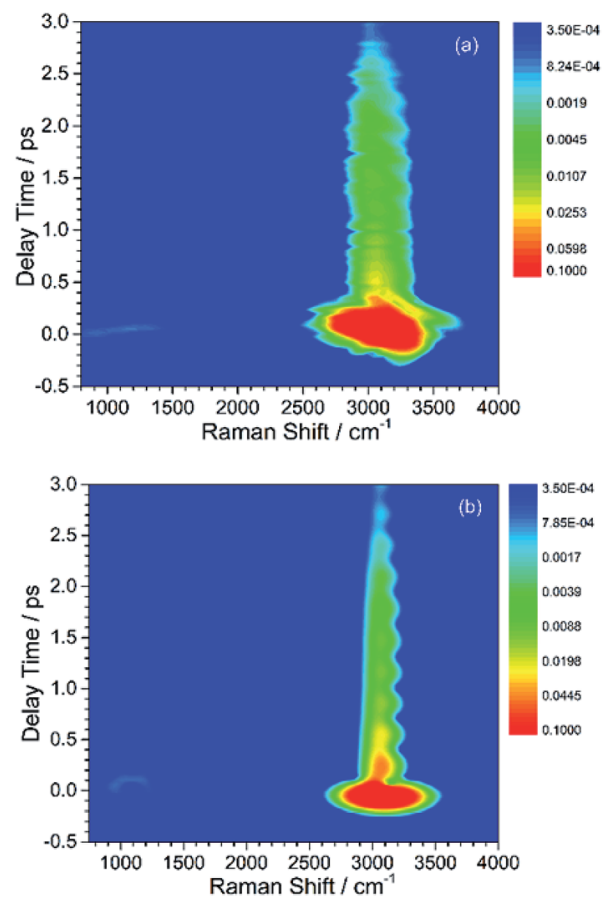

Fig. 1 Contour plots of experimental (a) and simulated (b) CARS spectra of liquid benzene. The slowly decayed CARS signal around $3000 \mathrm{~cm}^{-1}$ belongs to the directly excited parent modes, whose energy subsequently transfers to the daughter modes shown as the weak and quickly decayed signal around $1000 \mathrm{~cm}^{-1}$. All of plots are on logarithmic scales. 
show up in the simulated and experimental pattern. For comparison, the intensity maxima of the simulated and the experiment spectrum are normalized to 1 . Besides the electronic contributions at zero delay time, the other features all have comparable magnitudes, as shown in Fig. 1(a) and (b).

The peak intensities in the low-frequency region around $\sim 1000 \mathrm{~cm}^{-1}$, as a result of coherent vibrational energy transfer, are far lower than that in the high-frequency region. The part of the low-frequency region of both experimental and simulated contour map is redrawn in Fig. 2(a) and (b) for clarity. Two spectral components with a time difference of about 65 fs appear in both the two contour maps. The component around zero delay time originates from the coupling of $v_{4}$ and $v_{5}$, while the other component at around 65 fs delay time originates from the interference of $v_{3}$ and $v_{4}{ }^{9}$ The time difference of $65 \mathrm{fs}$ originates from the phase difference among these three daughter modes. One may notice that there are some differences in position and bandwidth between the simulated and the experimental results. There are two main reasons for the differences. On one hand, in principle, only Raman-active vibrations can be observed in CARS experiment, so only three vibrational modes around $1000 \mathrm{~cm}^{-1}$ with obvious Raman activity were taken into account in the simulation. But liquid benzene has a fundamental vibration $\left(\sim 1350 \mathrm{~cm}^{-1}\right)^{21}$ and some sum frequency vibrations with no Raman activity. In the case when IVR occurs, these vibrational modes around $1300 \mathrm{~cm}^{-1}$ will take part in and interference with the polarization of the $1181 \mathrm{~cm}^{-1}$ mode, which will makes a blueshift of the signal at about $65 \mathrm{fs}$ and shows a broadening
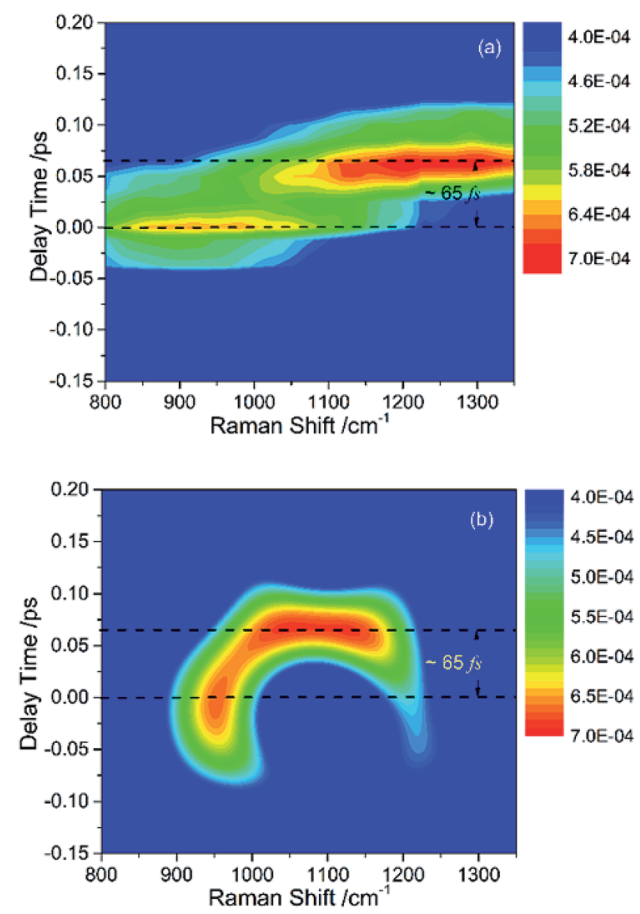

Fig. 2 Partial enlargement display of the CARS spectra in lowfrequency region, where the signal consists of (a) experimental result and (b) simulated result. The intensities of peak have comparable magnitudes. appearance. But we cannot determine which vibrational mode it is and its coupling with other oscillators, so we did not add any vibration mode in the simulation process. On the other hand, the simulation conditions are relatively ideal compared
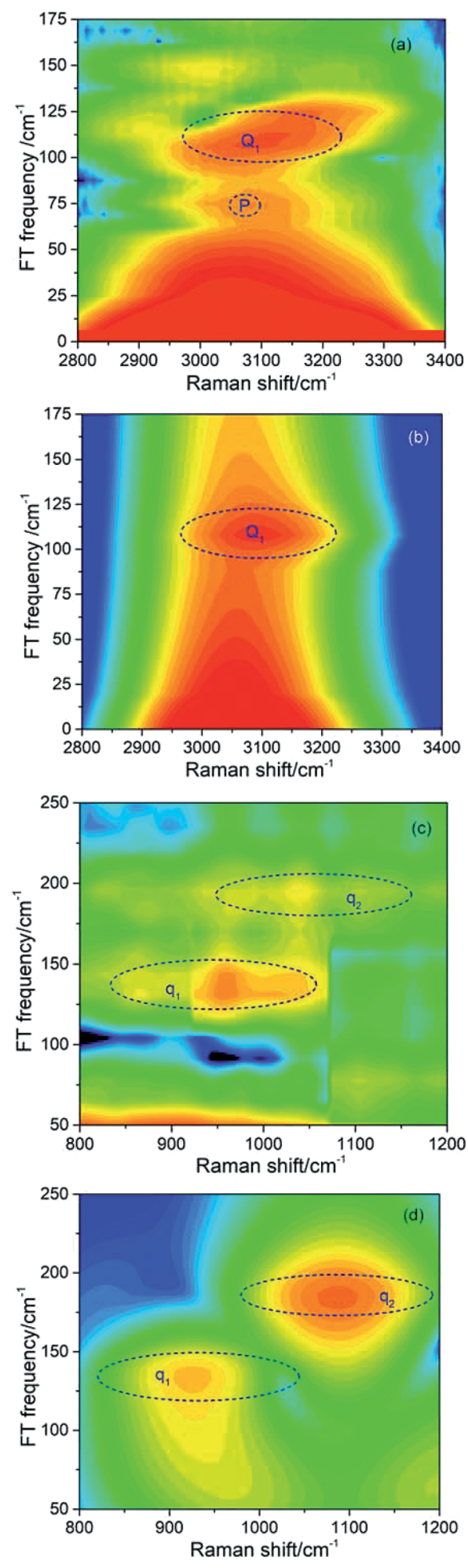

Fig. 3 Fourier transform (FT) spectra of CARS in different frequency regions. (a) and (c) show the FT spectra from experimental results, and (b) and (d) are the corresponding simulated results. 
with the experimental conditions. First, the laser in the experiment is not the transform limit pulse as used in the simulation. The spectral width of the pulses in experiment is actually wider than that used in the simulation. Second, the tilt of the signal around $1000 \mathrm{~cm}^{-1}$ reflects the chirp of the laser pulse, which is also not taken into account in the simulation. All of these factors will influence the pattern of vibrational spectrum. The phase fluctuation and/or nonideal signal-to-noise ratio in the experiment could also result in distortion of spectral pattern in the time domain. But for all this, the simulation still reproduces the main features of the experimental results.

(b) Spectra in frequency domain. In order to further confirm the reliability of the simulation, the simulated and experimental spectra are also compared in the frequency domain. The spectra in frequency domain are obtained by Fourier transform of the time-domain spectra, and the corresponding experimental and simulated results are shown in Fig. 3. The simulated spectra in both high- and low-frequency regions are consistent with the experimental results. In the high-frequency region, a peak marked by $\mathrm{Q}_{1}$ located at about $108 \mathrm{~cm}^{-1}$ appears in both the experimental and simulated FT spectra as show in Fig. 3(a) and (b). In the low-frequency region, two distinct coherent peaks marked by $\mathrm{q}_{1}$ and $\mathrm{q}_{2}$ appear in the simulated FT power spectra as show in Fig. 3(d). In Fig. 3(c), although the quality of the experimental spectrum is poor due to the low intensity of daughter modes, two coherent peaks can also be identified at the same locations. In the frequency-domain spectrum, the coherent peak usually comes from the interference of two adjacent vibrational modes, whose beat frequency is the frequency difference between the two modes. The origins of the coherent peaks labelled as $\mathrm{Q}_{1}, \mathrm{q}_{1}, \mathrm{q}_{2}$ are listed in Table 3 .

It should be noted that a peak marked by $\mathrm{P}$ located at about $70 \mathrm{~cm}^{-1}$ appears in the experimental FT spectrum in Fig. 3(a), but there is no corresponding peak in simulated spectrum. This peak arises from an intermolecular collective mode as reported by Shohei Kakinuma, ${ }^{22}$ which is not considered in the present simulation model.

From the above comparisons, it can be seen that the simulated CARS spectra well reproduce the experimental spectra. The reliability of the simulation results is demonstrated in both time and frequency domains. Although the simulation is carried out based on a simple coupled oscillator model, it can reasonably describe some features about the coupling between vibrational modes and between electron and vibrational modes.

Table 3 Assignment of the coherent peaks in the experimental and simulated FT spectra

\begin{tabular}{lll}
\hline Coherent peak & Beat frequency/cm & The modes involved \\
\hline $\mathrm{Q}_{1}$ & 109 & $v_{2}$ and $v_{6}$ \\
$\mathrm{q}_{1}$ & 137 & $v_{4}$ and $v_{5}$ \\
$\mathrm{q}_{2}$ & 189 & $v_{3}$ and $v_{4}$
\end{tabular}

\section{Discussion}

\subsection{Electron-vibration coupling}

In the previous experimental work, ${ }^{9}$ the appearance of vibrational signals in the low-frequency region was attributed to the vibrational coupling between parent modes and daughter modes. In fact, since the vibrational modes are surrounded by the electron, the IVR process should also be affected by electron. However, it is difficult to directly obtain the electronic contribution from the experimental spectra alone. The theoretical simulation introduced in this paper is really much helpful for analyzing the contribution of electron in IVR process.

Since the intensity of the daughter mode is a landmark of IVR process, it can reflect the effect of the electron-vibration coupling on the IVR process. The influence of electroncoupling on IVR can be preliminarily estimated by considering the two cases when all the electron-vibration coupling parameters are set to be zero and when all the vibrational coupling parameters are set to be zero. Fig. 4(a) shows the result of considering the vibrational coupling but ignoring the electron-vibration coupling. Although the signal maintains the similar pattern as Fig. 2(b), the intensity of the signal is two orders of magnitude lower. Fig. 4(b) is the result of only considering the electron-vibration coupling but ignoring the vibrational coupling. Compared with Fig. 2(b), the signal intensity is slightly lower, but the pattern has changed significantly. When only the electron-vibration coupling is considered,
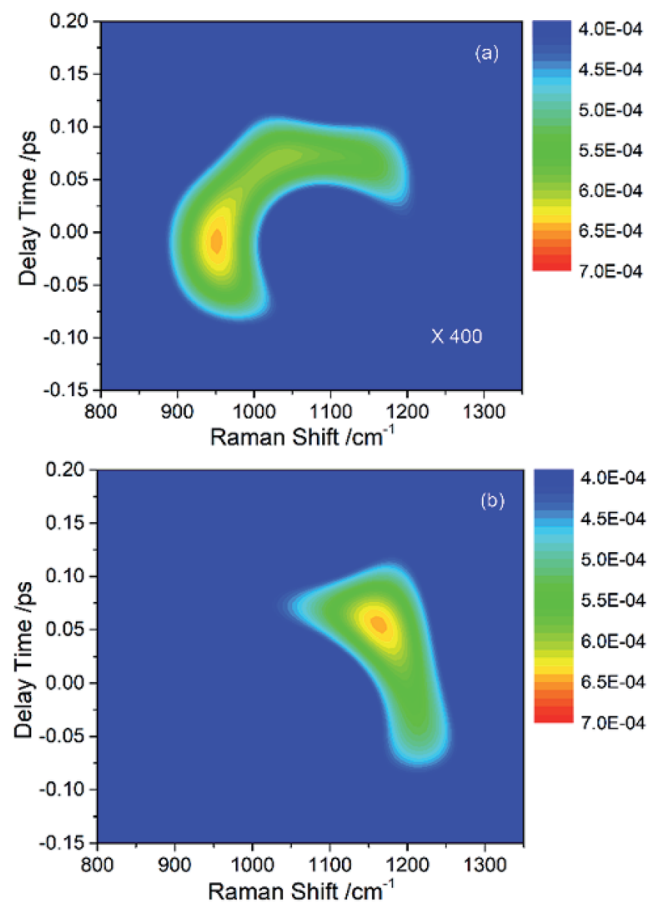

Fig. 4 (a) The simulated signal of daughter modes only considering the vibrational coupling but ignoring the electron-vibration coupling. The intensity of this signal is two orders of magnitude lower than that in Fig. 2(b). (b) The simulated signal of daughter modes only considering the electron-vibration coupling but ignoring the vibrational coupling. The pattern is distorted severely comparing with Fig. 2(b). 
energy can transfer to the daughter modes without the vibrational coupling, but not all the other vibrational modes can get energy from the parent modes. It can also be seen from the experimental CARS spectrum in Fig. 1 that only a few lowfrequency daughter modes coupled with $\mathrm{C}-\mathrm{H}$ stretching vibrations, while the absence of other modes outside of daughter mode region indicates that electron is not coupled with them or the coupling strength is very week. Thus, electron seems not to equally couple with all vibrations. We also found that a simple superposition of the two results shown in Fig. 4 could not reproduce the same signal pattern as that shown in Fig. 2(b). Therefore, in the IVR process, electron-vibration coupling and vibration-vibration coupling are not two isolated mechanisms. Electron will be involved in the vibrational mode coupling, and significantly changes the efficiency of vibrational energy transfer. This means that the electron, acting as a mediator, plays a key role in the coupling between the parent and daughter vibrational modes.

\subsection{The coupling strength}

In this coupled oscillator model, $K_{i j}$ is employed to represent the coupling strength between different oscillators, as expressed in eqn (1). Comparing with the experimental result, when the simulation result matches best with the experimental result, the coupling strengths between different oscillators can be obtained, as listed in Table 4. Even though the experimental signal-to-noise ratio of daughter modes is not good, the values of coupling strength cannot be precisely determined, but the magnitudes for various coupling can still be obtained.

Firstly, we consider the coupling between different vibrational modes. The coupling between $v_{1}$ and $v_{4}$ causing the vibrational energy transfer originates from their same symmetry, which is the same case for $v_{2}$ and $v_{3}$. Though the symmetries of $v_{4}$ and $v_{5}$ are different, the vibrational energy transfer also occurs. As the frequency difference between the two vibrational modes is very small, we deduce that the vibrational energy transfer happens with the help of intermolecular collective modes. It should be noticed that the frequency difference is almost twice the frequency of the collective mode mentioned in Section 3.2(b). Hence it is reasonable to infer that this coupling between $v_{4}$ and $v_{5}$ is related to the participation of the intermolecular collective mode. The simulation, which well reproduces the experimental CARS spectrum of benzene, confirms the reasonability of the parameter setting of vibrational coupling.

Secondly considering the contribution of electron, it is clear that the couplings between electron and different vibrational

Table 4 The coupling strength between different oscillators obtained from the simulation, the unit of $\mathrm{J} \mathrm{m}^{-1}$. ${ }^{2}$ Here, $K_{i j}=\sqrt{m_{i} m_{j}} \varpi_{i j}{ }^{2}$, refer to Table 2

\begin{tabular}{cccccccc}
\hline$K_{14}$ & $K_{23}$ & $K_{45}$ & $K_{\mathrm{e} 1}$ & $K_{\mathrm{e} 2}$ & $K_{\mathrm{e} 3}$ & $K_{\mathrm{e} 4}$ & $K_{\mathrm{e} 5}$ \\
\hline 0.61 & 0.18 & 0.19 & 0.08 & 0.03 & 0.21 & 0.80 & 0.003
\end{tabular}

modes are different. Considering the symmetry, electron will couple with the vibrational modes with $\mathrm{A}_{1 \mathrm{~g}}\left(v_{1}\right.$ and $\left.v_{4}\right)$ and $\mathrm{E}_{2 \mathrm{~g}}$ $\left(v_{2}\right.$ and $\left.v_{3}\right)$ symmetry for benzene. ${ }^{23}$ The coupling strength between electron and $v_{5}$ is very weak. The value 0.003 listed in Table 3 is the maximum possible value, and any value below 0.003 will hardly influence the simulated result. So the coupling between electron and $v_{5}$ is small enough to be neglected, which is reasonable considering the symmetry. There is another experimental phenomenon that no signals from other vibrational modes with $\mathrm{E}_{2 \mathrm{~g}}$ symmetry were observed in the experiment, which means electrons does not couple other $E_{2 g}$ symmetric vibrations of benzene. It is because, in the IVR process observed by CARS, electron only couples and couples simultaneously with the parent and daughter modes who have the same symmetry and the vibrational energy transfer between whom is permitted. This can be proved from the simulation result shown in Fig. 4 that the signal intensity of daughter modes will be two orders of magnitude greater when electronic contribution is involved.

\section{Conclusions}

A coupled oscillator model is established in order to get further understanding about the vibrational energy transfer observed in previous CARS experiment of liquid benzene. In this model, the electronic contribution is treated as an oscillator, and both molecular vibrational coupling and electronic and molecular vibration coupling are involved. The numerical simulated results well reproduce the experimental results, confirming the reliability of the simulation. The simulation results show that, there is strong coupling between the molecular vibrations with the same symmetry, which is in agreement with the experimental results. Beyond this, electron is found to play a mediator role in the IVR process through the theoretical simulation. When energy transfer occurs between two vibrational modes with the same symmetry, electron couples with the two modes simultaneously, which significantly increases the energy transfer efficiency.

\section{Conflicts of interest}

There are no conflicts to declare.

\section{Acknowledgements}

This work was supported by the National Natural Science Foundation of China (Grant No. 21673211), and the Science Challenging Program (Grant No. TZ2016001).

\section{Notes and references}

1 D. J. Nesbitt and R. W. Field, J. Phys. Chem., 1996, 100, 12735-12756.

2 B. C. Pein and D. D. Dlott, J. Phys. Chem. A, 2014, 118, 965973.

3 B. C. Pein, Y. Sun and D. D. Dlott, J. Phys. Chem. B, 2013, 117, 10898-10904. 
4 L. K. Iwaki, J. C. Deàk, S. T. Rhea and D. D. Dlott, Chem. Phys. Lett., 1999, 303, 176-182.

5 E. L. Chronister and D. D. Dlott, J. Chem. Phys., 1983, 79, 5286-5291.

6 A. M. Brodnikovsky, S. M. Gladkov and N. I. Koroteev, Opt. Commun., 1982, 40, 312-316.

7 M. Lütgens, S. Chatzipapadopoulos and S. Lochbrunner, Opt. Express, 2012, 20, 6478-6487.

8 X. Du, M. Zhang, X. He, Q. Meng, Y. Song, Y. Yang and J. Han, Chin. Phys. B, 2011, 20, 126301.

9 X. Liu, W. Zhang, Y. Song, G. Yu, Z. Zheng, Y. Zeng, Z. Lv, H. Song and Y. Yang, J. Phys. Chem. A, 2017, 121, 4948-4952.

10 X. Liu, Y. Song, W. Zhang, G. Zhu, Z. Lv, W. Liu and Y. Yang, RSC Adv., 2018, 8, 29775-29780.

11 D. C. Urbanek and M. A. Berg, J. Chem. Phys., 2007, 127, 044306.

12 W. M. Tolles, J. W. Nibler, J. R. Mcdonald and A. B. Harvey, Appl. Spectrosc., 1977, 31, 253-271.

13 H. U. Stauffer, J. D. Miller, M. N. Slipchenko, T. R. Meyer, B. D. Prince, S. Roy and J. R. Gord, J. Chem. Phys., 2014, 140, 024316.
14 K. Niu and S. Y. Lee, J. Chem. Phys., 2012, 136, 064504.

15 F. M. Kamga and M. G. Sceats, Opt. Lett., 1980, 5, 126-128.

16 A. S. Barker JR and R. Loudon, Rev. Mod. Phys., 1972, 44, 1847.

17 J. R. Ray, Am. J. Phys., 1973, 41, 1188-1190.

18 H. R. Martens, Simulation, 1969, 12, 87-94.

19 D. S. N. Parker, R. S. Minns, T. J. Penfold, G. A. Worth and H. H. Fielding, Chem. Phys. Lett., 2009, 469, 43-47.

20 M. J. Frisch, G. W. Trucks, H. B. Schlegel, G. E. Scuseria, M. A. Robb, J. R. heeseman, J. A. Montgomery, et al., Gaussian 03, Revision E.01, Gaussian Inc., Wallingford, CT, 2004.

21 C. H. Nicholas, E. M. Paul, D. A. Roger, S. A. Jamie, W. M. Christopher and J. L. Gregory, Chem. Phys. Lett., 1992, 197, 506-515.

22 S. Kakinuma and H. Shirota, J. Phys. Chem. B, 2015, 119, 4713-4724.

23 C. B. Duke, N. O. Lipari and L. Pietronero, Chem. Phys. Lett., 1975, 30, 415-420. 